\title{
Implementación de Sistema Óptico para Grabado de Redes de Bragg en Fibra Óptica
}

Nélida A. Russo ${ }^{(1) *}$, Sergio B. Noriega ${ }^{(1,2)}$ y Ricardo Duchowicz ${ }^{(1,2)}$

(1) Centro de Investigaciones Ópticas (CONICET La Plata - CIC), CC 124, (1900)

La Plata-Argentina. (e-mail: nelidar@ciop.unlp.edu.ar)

(2) Facultad de Ingeniería, Universidad Nacional de La Plata, Calle 1 y 47 (1900) La PlataArgentina.

* Autor a quien debe ser dirigida la correspondencia

Recibido Jun. 04, 2010; Aceptado Jul. 28, 2010; Versión Final recibida Oct. 12, 2010

\section{Resumen}

En este trabajo se presenta la implementación de un sistema óptico para el grabado de redes de Bragg en fibra óptica, empleando la técnica de la máscara de fase. El método consiste en irradiar una fibra fotosensible con un patrón de intensidades adecuado de luz UV que genera una modulación espacial del índice de refracción del núcleo de la fibra y forma la red de Bragg. Como fuente de iluminación se utiliza un láser pulsado de Nd-YAG cuadruplicado en frecuencia, con emisión en $266 \mathrm{~nm}$. Se describe el sistema desarrollado, se analizan los resultados y se mencionan aplicaciones desarrolladas con las redes fabricadas.

Palabras clave: fibra óptica, redes de Bragg en fibra, máscara de fase, láser de Nd-YAG

\section{Optical System Development for Fiber Bragg Grating Writing}

\begin{abstract}
This work presents the implementation of an optical system for fiber Bragg grating writing by using the phase mask technique. The method consists of radiating a photosensitive optical fiber with a suitable intensity pattern of UV light that generates a spatial modulation of the fiber core refraction index and creates the Bragg grating. As light source a beam of an Nd-YAG pulsed laser, of quadrupled frequency, and $266 \mathrm{~nm}$ emission, was used. The proposed system is described, the results are analyzed, and applications developed with fiber Bragg grating are mentioned.
\end{abstract}

Keywords: optical fibers, fiber Bragg gratings, phase mask, Nd-YAG laser 


\section{INTRODUCCIÓN}

En la actualidad las redes de Bragg grabadas en fibra óptica (FBG) son ampliamente utilizadas en la fabricación de diferentes tipos de dispositivos empleados tanto en el área de las comunicaciones ópticas como en el ámbito industrial. Entre sus principales aplicaciones pueden mencionarse filtros sintonizables para multiplexado en longitud de onda, ecualizadores de ganancia en amplificadores ópticos, compensadores de dispersión, estabilizadores en longitud de onda de la emisión de láseres semiconductores, procesamiento fotónico de señales, tomografía óptica coherente para generación de imágenes biomédicas, etc. (Preciado et al., 2007; Dai et al., 2006; Capmany et al., 2005; Choi et al. 2005). También son empleadas como elementos de realimentación de la cavidad en el desarrollo de láseres continuos o pulsados de fibra óptica (Andrés, et al., 2008). Asimismo, son extensamente utilizadas en aplicaciones de sensado remoto que incluyen el monitoreo de estructuras civiles (puentes, edificios, carreteras, etc.), el testeo no destructivo en procesos de fabricación, la evaluación de diferentes parámetros en la industria automotriz y aeroespacial (esfuerzo, temperatura, presión, vibraciones, desplazamiento, etc.), en ambientes peligrosos o corrosivos (pozos de extracción de petróleo, industria petroquímica, etc.), entre otras (Optical-fibre sensors, en Nature Photonics, 2008). Los sensores basados en redes de Bragg grabadas en fibra óptica, son considerablemente sensibles y traducen directamente el parámetro sensado en variaciones de sus características espectrales.

Dichas redes consisten en una modulación periódica o aperiódica del índice de refracción efectivo del núcleo de una fibra óptica, generada a lo largo de la misma al irradiarla con un patrón apropiado de luz ultravioleta. Cuando un haz de luz de cierto ancho espectral se propaga en la fibra y alcanza la red de Bragg, una banda espectral muy angosta (centrada en la longitud de onda de Bragg, $\lambda_{\text {Bragg }}$ ) es reflejada, mientras que las demás componentes espectrales la atraviesan sin sufrir modificación, lo que permite utilizarlas como filtro muy selectivo en longitud de onda. El contraste de la variación de índice de refracción, el período de la modulación y la longitud de la red, determinan sus propiedades de transmisión y reflexión. Entre las ventajas de las FBG sobre otras tecnologías se pueden mencionar la geometría "todo-fibra", la baja pérdida de inserción, la alta pérdida de retorno y fundamentalmente, la flexibilidad que ofrecen para alcanzar características espectrales deseadas.

Existen diferentes métodos para fotoinducir redes en fibra óptica fotosensible, los cuales básicamente pueden agruparse en dos categorías: de escritura interna o externa (Kashyap, 2010; Othonos y Kalli, 1999). Aunque el descubrimiento de la fotosensibilidad en fibras ópticas data de 1978, cuando Hill et al. detectaron la formación de cambios periódicos permanentes en el índice de refracción del núcleo (dopado con $\mathrm{Ge}$ ) de una fibra óptica cuando en la misma se acoplaba el haz proveniente de un láser de ión argón con emisión en $488 \mathrm{~nm}$, ciertas limitaciones hicieron decaer el interés de las investigaciones en este tema. La técnica (considerada de escritura interna) se basaba en la modificación autoinducida del índice de refracción a través de un proceso de absorción de dos fotones. Sin embargo, las redes obtenidas sólo podían operar en una longitud de onda de Bragg coincidente con la de grabado. La demostración de la técnica de escritura lateral (Meltz et al., 1989) atrajo nuevamente la atención hacia la generación de redes de Bragg en fibra. Estos autores propusieron la técnica interferométrica de fabricación, en la que se divide la luz UV de entrada en dos haces que luego son recombinados para generar un diagrama de interferencia que irradia lateralmente a la fibra e induce una modulación del índice de refracción en el núcleo de la misma. El ángulo entre los haces permite controlar el período del patrón de interferencia y por lo tanto, la longitud de onda de Bragg o de máxima reflexión. Suelen utilizarse interferómetros que dividen la amplitud del haz o el frente de onda, pero se requiere que la fuente láser posea suficiente coherencia temporal y buena coherencia espacial. Además de esta técnica de escritura externa, existen otras como la grabación "punto por punto" y la técnica de la máscara de fase, las cuales permiten obtener redes de Bragg más versátiles. La técnica de fabricación "punto por punto" consiste en enfocar lateralmente un único pulso de radiación UV dentro del núcleo de la fibra óptica, de modo de modificar localmente el índice de refracción (Malo et al., 1993). La fibra es luego trasladada en la dirección de su eje, una distancia correspondiente al período de la red y nuevamente irradiada. Repitiendo este proceso se logra formar la estructura de la red de Bragg en el núcleo de la fibra. Es esencial en esta técnica disponer de un sistema 
submicrométrico de traslación estable y preciso. Este esquema de grabación es particularmente apropiado para fabricar redes de período largo $\left(\Lambda_{\text {red }}>100 \mu \mathrm{m}\right)$.

Uno de los métodos más efectivos para inscribir redes de Bragg en fibra fotosensible es la técnica de la máscara de fase (Hill et al., 1993; Othonos y Lee, 1995; Yeh et al., 2003). La ventaja fundamental de este procedimiento radica en la sencillez del montaje experimental utilizado, que no requiere la estabilidad mecánica necesaria en los interferómetros implementados con elementos discretos, ni las exigentes prestaciones del sistema de posicionamiento y traslación que debe emplearse para grabar redes punto a punto. Esta técnica emplea un elemento óptico difractivo (la máscara de fase, básicamente una red de difracción que funciona por transmisión) para modular espacialmente el haz UV de escritura. Al irradiar dicho elemento con un único haz UV (típicamente en la región espectral 240-260 nm, donde el proceso de fotosensibilización en fibras ópticas es más eficiente) (Jensen et al. 2001; Kashyap, 2010), la luz difractada por los dos primeros órdenes forma al interferir, un patrón de intensidad periódico y de alto contraste que fotoimprime la modulación de índice de refracción del núcleo de la fibra óptica. El contraste de esta distribución de intensidades es fuertemente dependiente de la longitud de coherencia del haz por tratarse de un fenómeno interferométrico (Prakash et al., 2006). Las fuentes láser de emisión continua poseen una mayor longitud de coherencia que las pulsadas, lo que permite obtener redes con mayores profundidades de modulación de índice y, por consiguiente, mayores reflectividades. Del mismo modo, una mejora en la coherencia espacial del haz da como resultado una mayor tasa de crecimiento de la reflectividad de la red grabada. Actualmente se han desarrollado sistemas más complejos de grabado de redes de Bragg en fibra que utilizan la técnica de la máscara de fase en conjunción con fuentes de pulsos ultracortos (fs) (Zagorul'ko et al., 2003; Mihailov et al., 2004). Este tipo de láseres no dañan las fibras a pesar de su alta intensidad debido a la extremadamente corta duración del pulso luminoso, pero son equipos sofisticados y de un alto costo.

En este trabajo se presenta la implementación de un sistema sencillo para realizar el grabado de FBG empleando la técnica de la máscara de fase y utilizando como fuente de luz UV, la radiación generada en $266 \mathrm{~nm}$ por un láser pulsado de Nd-YAG cuadruplicado en frecuencia. Dado que las redes de Bragg grabadas en fibra óptica constituyen un elemento de suma importancia en el diseño y desarrollo de novedosos dispositivos fotónicos para el procesado óptico de señales en sistemas de comunicaciones, así como para el sensado de diferentes parámetros de aplicación en las industrias automotriz, aeroespacial, de ingeniería civil, etc., resulta de suma importancia la implementación de una estación de grabado de las mismas que permita su diseño y fabricación con vistas a generar nuevas aplicaciones de estos elementos. Además, por los motivos antes mencionados, se trata de una tecnología que posee un impacto relevante en el desarrollo de los países de América Latina. En particular, las actividades de investigación llevadas a cabo en nuestro Laboratorio están destinadas a la generación de nuevos dispositivos totalmente implementados en fibra óptica. El objetivo final es lograr un sistema versátil que permita de una manera flexible la fabricación de redes diseñadas con las características espectrales adecuadas según el tipo de aplicación, y de calidad comparable a las producidas comercialmente en algunos países desarrollados.

\section{PRINCIPIO DE OPERACIÓN}

Cuando un haz luminoso UV incide sobre una máscara de fase, éste es difractado en varios órdenes $m=0, \pm 1, \pm 2, \ldots$. Los órdenes incidente y difractados satisfacen la siguiente condición:

$A_{p m}=\frac{m \lambda_{w}}{\left(\sin \frac{\theta_{m}}{2}-\sin \theta_{l}\right)}$

donde $\Lambda_{p m}$ es el período de la máscara de fase, $\lambda_{u v}$ es la longitud de onda del haz UV incidente, $\theta_{m} / 2$ es el ángulo del orden $m$ difractado y $\theta_{i}$ es el ángulo de incidencia sobre la máscara. Si el haz UV incide perpendicularmente, los órdenes difractados son solamente $m=0$ y \pm 1 . Habitualmente las máscaras de fase se diseñan de modo de minimizar el orden 0 . De este modo, la máscara de 
fase actúa como una red de difracción de precisión, cuyos dos haces de salida crean un patrón de interferencia en la región del espacio donde se solapan.

El período de la máscara de fase se relaciona con el período de la modulación de índice generada en la fibra $\left(\Lambda_{\text {red }}\right)$ y con la longitud de onda de Bragg $\left(\lambda_{\text {Bragg }}\right)$ de la siguiente manera:

$\Lambda_{\text {red }}=\frac{\Lambda_{p m}}{2}=\frac{\lambda_{\text {Eragg }}}{2 n_{\text {eff }}}$

donde $n_{\text {eff }}$ es el índice de refracción efectivo del núcleo de la fibra. Así por ejemplo, una red de Bragg diseñada para operar en la región de $\sim 1.5 \mu \mathrm{m}$ tendrá un período de unos $500 \mathrm{~nm}$.

Centrándonos en los órdenes -1 y +1 , a la salida de la máscara se forma un patrón de interferencia normal a la misma cuya periodicidad es independiente de la longitud de onda del haz láser UV incidente. Colocando una fibra fotosensible detrás y en contacto con la máscara, y orientando sus corrugaciones perpendicularmente al eje de la fibra, se grabará sobre el núcleo la modulación deseada del índice de refracción (FBG) como se observa en la Figura 1. El patrón grabado dentro de la fibra es una copia escalada (en cuanto al período y "chirp") de la máscara de fase utilizada.

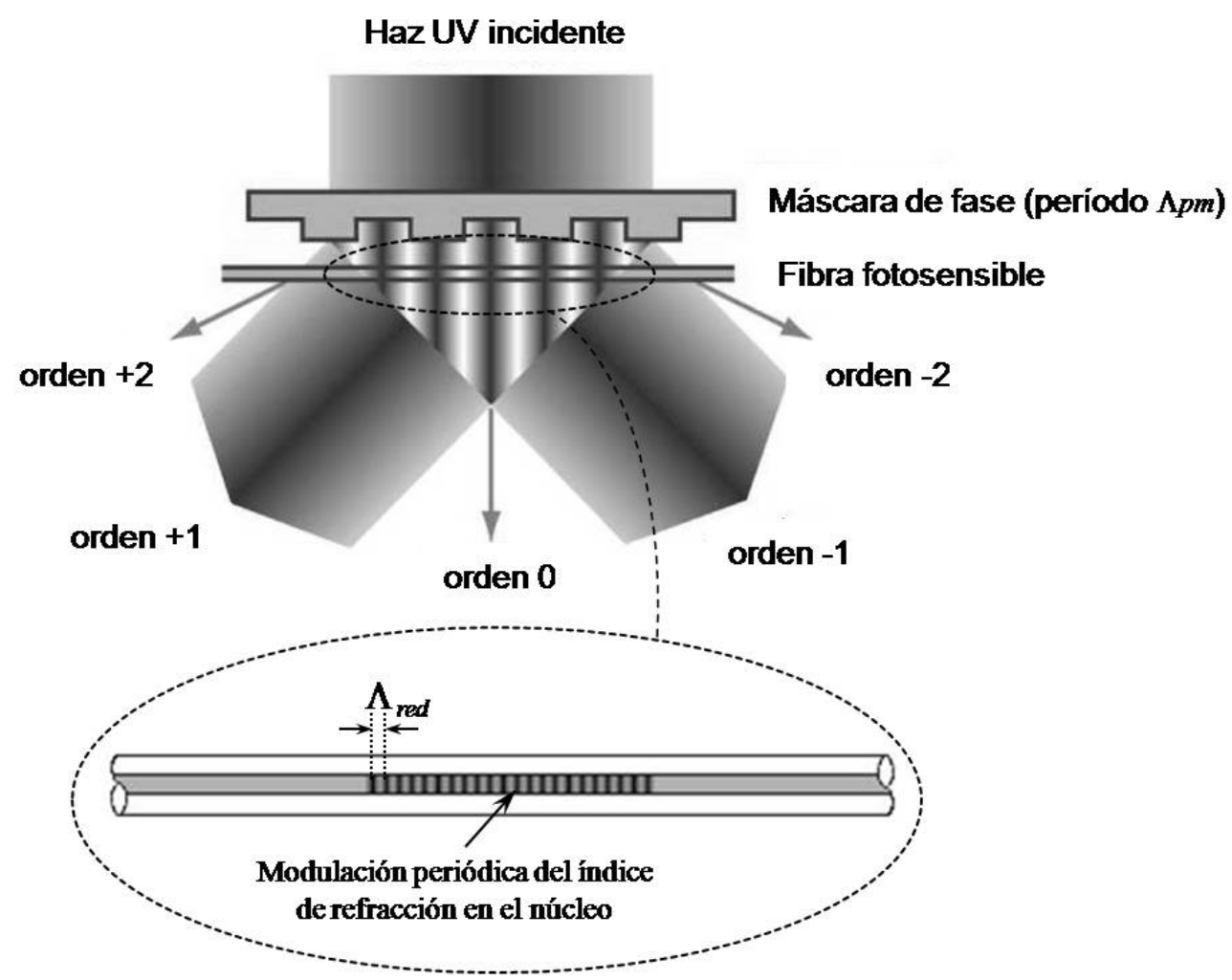

Fig. 1: Diagrama esquemático del proceso de grabado de FBG con máscara de fase

\section{DESCRIPCIÓN DEL SISTEMA IMPLEMENTADO}

El esquema experimental se muestra en la Figura 2. La fuente de UV es un láser de Nd-YAG capaz de entregar pulsos de $650 \mathrm{~mJ}$ en $1064 \mathrm{~nm}$, con una frecuencia de repetición máxima de 10 $\mathrm{Hz}$. Con la ayuda de dos cristales dobladores de frecuencia se obtiene emisión en $266 \mathrm{~nm}$. Sin embargo, dado que la eficiencia de los cristales no es de $100 \%$, el haz de salida contiene algo de radiación infrarroja $(1064 \mathrm{~nm})$ y visible $(532 \mathrm{~nm}$, salida del primer doblador). Utilizando un prisma Pellin-Brocca es posible separar angularmente la energía contenida en las diferentes longitudes de onda, y utilizar sólo aquella porción correspondiente al UV. Luego de ser expandido y colimado, el haz incide sobre una lente cilíndrica montada sobre un microposicionador (MP1), generando una línea horizontal a la distancia focal de la lente, donde se coloca la máscara de fase. En 
contacto con la cara de la máscara por donde emerge el haz se coloca la fibra óptica fotosensible tratando de garantizar un apoyo homogéneo a lo largo de la red. Tanto la máscara como la fibra se encuentran montadas en un microposicionador X-Y (MP2) a fin de poder alinear el sistema y enfocar el haz sobre la máscara.

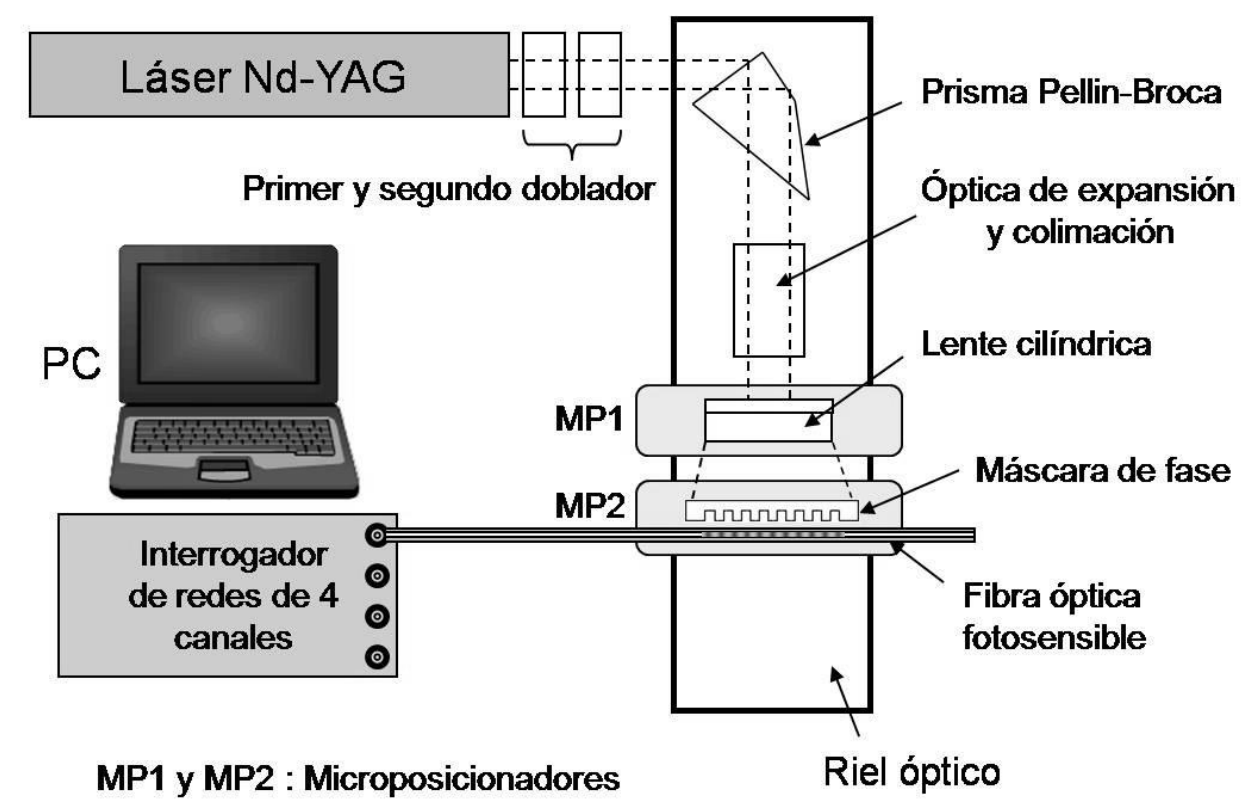

Fig. 2: Esquema experimental del sistema para grabado de redes de Bragg en fibra óptica

Para lograr el seguimiento del proceso de grabado se conecta un extremo de la fibra fotosensible (mediante una terminación FC-APC) a un interrogador de redes de 4 canales, de alta resolución y exactitud. A través de la conexión remota de este instrumento a una PC, se monitorea la evolución del grabado de la red observando en el monitor de la computadora el espectro por reflexión de la misma. Esta información in-situ es muy importante ya que da un panorama al instante de cómo se produce el proceso de grabado, permitiendo realizar si es necesario, los ajustes pertinentes a fin de optimizar dicha operación. Las características relevantes evaluadas fueron: ancho espectral, reflectividad máxima relativa, longitud de onda central, supresión de lóbulos laterales, etc. En la Figura 3 se muestran fotografías de la estación implementada para el grabado de redes de Bragg en fibra.
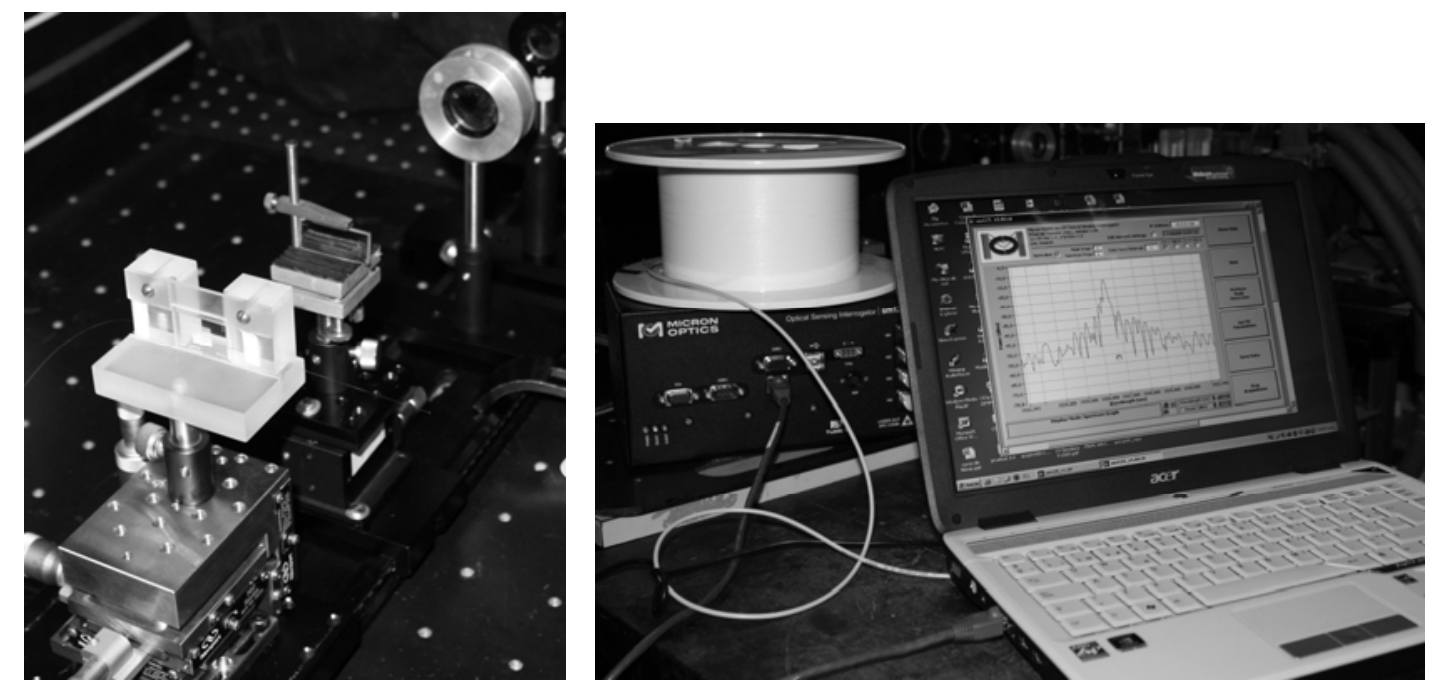

Fig. 3: Sistema de grabación implementado: (a) Parte del montaje óptico y (b) Monitoreo del proceso de grabado 
Las especificaciones de la fibra fotosensible empleada son: longitud de onda de operación 1500$1600 \mathrm{~nm}$, diámetro del campo modal $10.5 \mu \mathrm{m} @ 1550 \mathrm{~nm}$, apertura numérica 0.13, y diámetro del cladding $125 \mu \mathrm{m}$. La máscara de fase es de sílica fundida, diseñada para una longitud de onda de iluminación de $266 \mathrm{~nm}$, con un período uniforme de 1054,79 nm y se garantiza una supresión de orden cero menor al $4 \%$.

El interrogador de redes es un instrumental de medición cuya finalidad es caracterizar espectralmente diferentes componentes ópticos pasivos tales como FBG, sensores interferométricos, etc., permitiendo realizar tanto mediciones de longitud de onda como de potencia óptica. Está específicamente diseñado para la medición de fenómenos estáticos a moderadamente dinámicos. Incluye un láser de fibra sintonizable en la región 1510-1590 nm, y emplea un filtro óptico FabryPerot que permite obtener una emisión de muy bajo ancho espectral y lograr resoluciones en longitud de onda de $1 \mathrm{pm}$. Posee además un fotodetector de alta sensibilidad y rango dinámico. Puede operarse remotamente desde una PC vía una interface Ethernet y emplea una utilidad basada en un entorno LABVIEW, que permite la visualización del espectro de reflexión del dispositivo bajo monitoreo.

\section{RESULTADOS Y DISCUSIÓN}

Como se mencionó en la sección anterior, la máscara de fase empleada tiene un período constante en toda su extensión por lo que las redes generadas resultan uniformes, salvo que se empleen modificaciones en el montaje u otra máscara apropiada para generar perfiles de índice de refracción más complejos.

Cuando el haz UV tiene una amplitud uniforme en toda la región iluminada de la máscara de fase, la modulación de índice que genera sobre la fibra también se mantiene constante sobre la zona irradiada y luego disminuye abruptamente a cero en ambos extremos. Esto hace que las redes obtenidas presenten una respuesta espectral como la mostrada en la Figura 4, en la que se observa la presencia de importantes lóbulos laterales, lo cual puede ser inapropiado para ciertas aplicaciones como por ejemplo en la implementación de filtros ópticos.

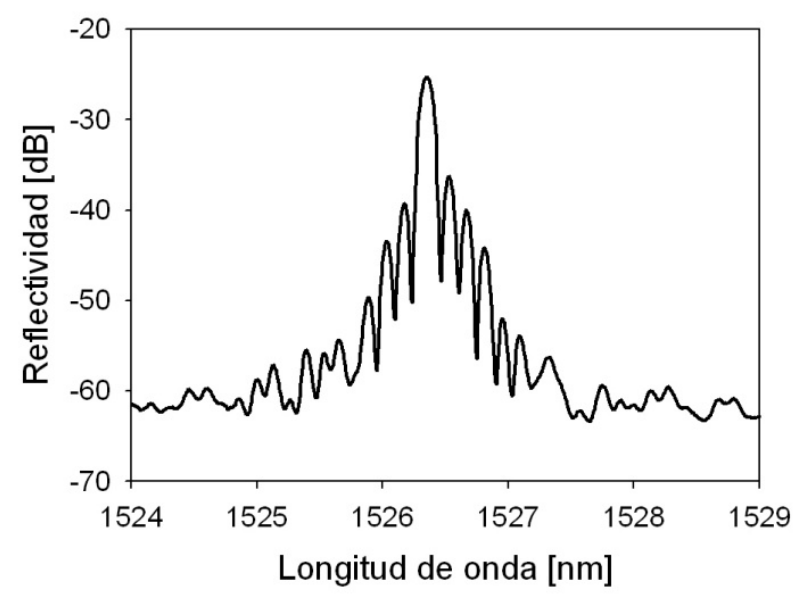

Fig. 4: Espectro de reflexión con lóbulos laterales

Con el objeto de remover o reducir sustancialmente estos lóbulos se aplicó una técnica conocida como apodización, que consiste en suavizar la variación de índice de refracción haciendo que su amplitud cambie gradualmente tanto en el comienzo como en el final de la red. Luego de acondicionar espacialmente el perfil del haz que incide sobe la máscara de fase (diafragmándolo y haciendo que la iluminación de la misma tenga un perfil aproximadamente gaussiano), se lograron obtener redes con espectros de reflexión y transmisión como los mostrados en las Figuras 5 a y b, 
respectivamente, donde la longitud de onda de máxima reflexión $\left(\lambda_{\text {Bragg }}\right)$ es de $1526.325 \mathrm{~nm}$ y su ancho espectral a $-3 \mathrm{~dB}$ es de $0.14 \mathrm{~nm}$.
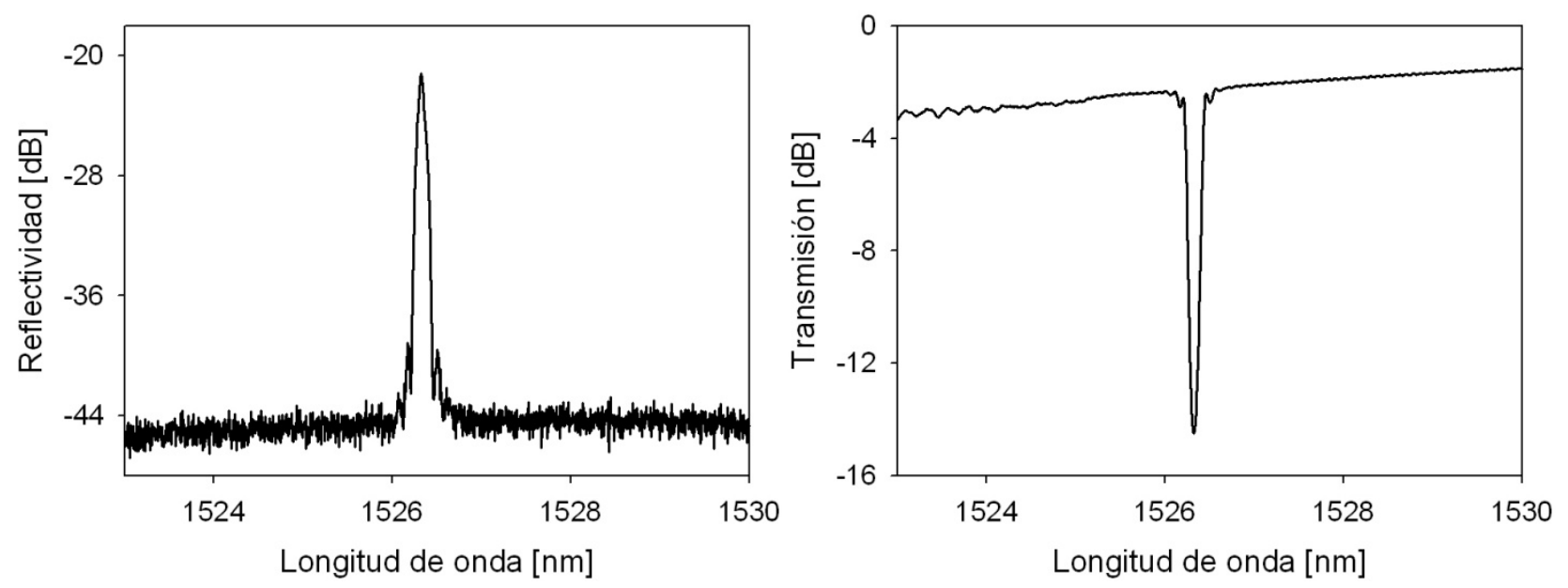

Fig. 5: (a) Reflectancia y (b) transmisión de una red de Bragg grabada en el laboratorio

Si la estabilidad del sistema de grabado es apropiada, es posible irradiar la fibra durante varios minutos con el objeto de aumentar la reflectividad máxima de las redes obtenidas. Sin embargo, la tasa de crecimiento es muy rápida inicialmente y luego tiende a una especie de saturación. Los tiempos habituales de grabado con nuestro sistema fueron de unos 10-15 minutos, dependiendo de la energía por pulso y la tasa de repetición que se seleccionase en el láser de Nd-YAG, con el objeto de no dañar la máscara de fase.

La irradiación con luz UV produce defectos localizados en el material fotosensible generando una variación del índice de refracción que, dependiendo de la intensidad del haz, oscila entre $10^{-5}$ y $10^{-}$ ${ }^{3}$. Habitualmente, la fotosensibilidad de las fibras de sílice es incrementada agregando dopantes como germanio, boro, fósforo, o cargando la fibra con hidrógeno previamente a su irradiación (Lemaire et al., 1993).

Dada la naturaleza interferométrica del método, cuanto menor sea la coherencia espacial de la fuente UV de iluminación empleada, más próxima a las corrugaciones de la máscara de fase deberá colocarse la fibra, para de esta manera lograr maximizar el contraste de la modulación de índice generada y por lo tanto, la reflectividad de la red grabada (Othonos y Lee, 1995; Dyer et al., 1995; Prakash et a., 2006). Considerando la longitud de coherencia como $L_{c}=\lambda^{2} / \Delta \lambda$, donde $\Delta \lambda$ es el ancho espectral de la fuente, $\lambda$ la longitud de onda de operación, y asumiendo $\lambda=266 \mathrm{~nm}$, $\Delta \lambda=0.6 \mathrm{~nm}$, resulta que la fibra deberá ubicarse a una distancia menor a $118 \mu \mathrm{m}$. Es decir, nuestra fibra de $125 \mu \mathrm{m}$ de diámetro de recubrimiento, debe colocarse en contacto con la máscara para que la distancia al núcleo cumpla con esa condición. Sin embargo, en un futuro inmediato nuestro Centro contará con una nueva fuente láser de Nd-YAG cuya longitud de coherencia es un orden de magnitud superior, lo que hará menos crítico este posicionamiento, asegurando un mejor contraste del patrón de iluminación, al tiempo de disponer de una distancia apropiada para generar perfiles más complejos, por ejemplo colocando la fibra con cierta inclinación respecto del plano de la máscara.

En las redes de Bragg uniformes, tanto el índice modal como el período de la red son constantes a lo largo del eje de propagación y por lo tanto, existe una única longitud de onda que cumple con la condición de Bragg, dada por la ecuación (2). Sin embargo, es posible modificar ligeramente (unos pocos $\mathrm{nm}$ ) la longitud de onda de máxima reflexión obtenida con una misma máscara de fase, tensionando la fibra durante el proceso de grabado, de modo de cambiar el período de la red. Otra alternativa más sofisticada involucra la recombinación de dos órdenes difractados por la máscara de fase $(0$ y +1 o -1 y +1$)$ mediante un par de espejos que permitan modificar el período del patrón 
de interferencia y por lo tanto, la longitud de onda de Bragg. En esta configuración, llamada interferómetro Talbot (Dyer et al., 1996), la fibra fotosensible ya no se encuentra en contacto con la máscara sino en un punto de enfoque en donde se hace interferir los haces.

La generación de redes no uniformes, donde el índice de refracción efectivo y/o el período de la red varía a lo largo de la dirección de propagación (Z), posee también múltiples aplicaciones. Las redes chirpeadas, en las que se induce una variación lineal o chirp en el período de la red, son utilizadas particularmente para compensar la dispersión cromática en enlaces de fibra óptica de alta velocidad, ya que permiten introducir un retardo dependiente de la longitud de onda (Litchinitser et al., 2007; Pérez-Millán et al., 2008).

Para mantener estables las propiedades ópticas de las FBGs por períodos de hasta 25 años, debe realizarse un recocido térmico a alta temperatura $\left(>250^{\circ} \mathrm{C}\right)$ después de la grabación. Las condiciones térmicas de recocido deben elegirse según el tipo de fibra fotosensible utilizada y según las condiciones de grabación de las redes (Cerri Triques et al., 2004).

Actualmente se está trabajando en la optimización del montaje con vistas a lograr la automatización del proceso de grabado. Así por ejemplo, se está diseñando un nuevo mecanismo de posicionamiento de la fibra fotosensible, independiente de la máscara de fase, lo que permitirá realizar un ajuste más preciso en la alineación, con la posibilidad de modificar mecánicamente la longitud de onda de grabado de las FBG, generar redes no-uniformes y grabar varias redes en una misma fibra (por ejemplo para implementar filtros tipo Fabry-Perot). Los parámetros de ajuste durante la grabación (tiempo de irradiación, energía y perfil del haz de iluminación, tasa de repetición de los pulsos UV, chirp rate de la máscara de fase, tensión mecánica aplicada sobre la red, "tilt" entre la fibra y la máscara, etc.) se seleccionan en función de las características espectrales deseadas.

\section{CONCLUSIONES}

La principal motivación para la implementación que se muestra en este trabajo radica en que las redes de Bragg grabadas en fibra óptica constituyen un elemento de suma importancia en el desarrollo de nuevos dispositivos fotónicos, y actualmente deben ser importadas desde el exterior dado que no existe ningún laboratorio de investigación o empresa que las fabrique en nuestro país. Con el objeto de lograr una mayor disponibilidad de estos componentes a menor costo y con la flexibilidad de poder diseñarlas con diferentes características espectrales en función de la aplicación y de grabarlas sobre distintos tipos de fibra (fotosensibles, dopadas con tierras raras, etc.), se implementó una estación de grabado de redes empleando la técnica de la máscara de fase. Esta técnica tiene la ventaja de reducir notoriamente la complejidad del sistema de grabado, proveyendo una forma simple, versátil, robusta e inherentemente estable de fabricar redes de Bragg. La flexibilidad de la técnica utilizada está nos permitiendo encarar un conjunto de aplicaciones como nuevas configuraciones de láseres pulsados en régimen "mode-locking" (Russo y Duchowicz, 2010) sensores de temperatura y de "strain" para la medición de la contracción polimérica experimentada por resinas de fotocurado empleadas en odontología (Russo et al., 2010), sensores para el monitoreo del estado (medición de temperatura en lugares específicos y control de daños estructurales) de vehículos lanzadores con control de vuelo, etc.

A pesar de que suelen utilizarse como haz de iluminación UV, láseres CW de ion-Argón con emisión en 244 nm, láseres pulsados de excimero KrF en 248 nm, o ArF en 193 nm, nosotros empleamos un láser pulsado de Nd-YAG cuadruplicado en frecuencia con emisión en $266 \mathrm{~nm}$, con el que cuenta nuestro Centro. Los resultados alcanzados permitieron corroborar la factibilidad de la utilización de este tipo de fuentes para el grabado de FBG, a pesar de la baja fotosensibilidad de la fibra en esta longitud de onda.

La evaluación de la calidad del producto obtenido se realizó mediante el análisis espectral a tiempo real, lo que permitió realizar los ajustes necesarios para optimizar las características del mismo. Del análisis de los resultados alcanzados puede inferirse que el sistema permite generar 
redes de una buena reflectividad y adecuado ancho espectral, así como un alto grado de repetibilidad de las redes fabricadas.

Respecto de los errores asociados con el sistema empleado, existe una serie de parámetros inherentes a la máscara de fase que influyen en las características espectrales de la red a grabar, tal como la exactitud y uniformidad del período de la máscara de fase, el grado de supresión del orden cero que ella genera, entre otras. Se está evaluando el grado en que estos errores y otros relativos al posicionamiento de los diferentes componentes del sistema, se traducen en la modificación de los parámetros deseados en la red grabada.

\section{AGRADECIMIENTOS}

Este trabajo fue apoyado por: CONICET (PIP 6156/05 y 112-200801-01769), Fac. Ingeniería UNLP (Proyecto I128), ANPCyT (PICT 2005 38289) y CIC (Res.730/07).

\section{REFERENCIAS}

Andrés M.V., J.L. Cruz, A. Díez, P. Pérez-Millán y M. Delgado-Pinar; Actively Q-switched all-fiber lasers, Las. Phys. Lett. 5 (2), 93-99 (2008).

Capmany, J., D. Pastor, B. Ortega, J.Mora y M.V. Andrés; Photonic processing of microwave signals, IEE Proc. Optoelectron. 152, 299-320 (2005).

Cerri Triques, A. L. y otros 6 autores; Thermal treatment of fiber Bragg gratings recorded using high power lasers, J. Microw. Optoelectron. 3 (5), 127-134 (2004).

Choi, E.S., J. Na, S. Young Ryu, G. Mudhana y B. Ha Lee; All-fiber variable optical delay line for applications in optical coherence tomography: Feasibility study for a novel delay line, Opt. Express 13 1334-1345 (2005).

Dai, Y., X. Chen, J. Sun y S. Xie; Wideband multichannel dispersion compensation based on a strongly chirped sampled Bragg grating and phase shifts, Opt. Lett. 31 (3), 311-313 (2006).

Dyer, P.E., R.J. Farley y R. Giedl; Analysis of grating formation with excimer laser irradiated phase masks, Opt. Comm. 115 (3-4), 327-334 (1995).

Dyer, P.E., R.J. Farley y R. Giedl; Analysis and application of a 0/1 order Talbot interferometer for $193 \mathrm{~nm}$ laser grating formation, Opt. Comm. 129 (1-2), 98-108 (1996).

Hill, K. O., Y. Fujii, D. C. Johnson y B. S. Kawasaki; Photosensitivity in optical fiber waveguides: Application to reflection filter fabrication, Appl. Phys. Lett. 32 (10), 647-649 (1978).

Hill, K. O., B. Malo, F. Bilodeau, D. C. Johnson y J. Albert; Bragg gratings fabricated in monomode photosensitive optical fiber by UV exposure through a phase mask, Appl. Phys. Lett. 62 (10), 10351037 (1993).

Jensen, J., P. Varming, B. Liu y W. Gries; Comparision of photosensitivity in Germanium doped Silica fibers using 244nm and 266nm continuous-wave lasers, Conference Paper WDD90, Optical Fiber Communication Conference (OFC), Anaheim, California, USA, 17 al 22 de Marzo (2001).

Kashyap, R.; Fiber Bragg Gratings (Second Edition), Cap. 2: Photosensitivity and Photosensitization of Optical Fibers, 15-51 y 53-118, Academic Press - Elsevier, USA (2010).

Lemaire, P. J., R. M. Atkins, V. Mizrahi y W. A. Reed; High pressure $\mathrm{H}_{2}$ loading as a technique for achieving ultrahigh UV photosensitivity and thermal sensitivity in $\mathrm{GeO}_{2}$ doped optical fibres, Electron. Lett. 29 (13), 1191-1193 (1993). 
Litchinitser, N. M., M. Sumetsky y P. S. Westbrook; Fiber-based tunable dispersion compensation, J. Opt. Fiber Commun. Rep. 4, 41-85 (2007).

Malo, B., K. 0. Hill, F. Bilodeau, D. C. Johnson y J. Albert; Point-by-point fabrication of micro-Bragg gratings in photosensitive fibre using single excimer pulse refractive index modification technique, Electron. Lett. 29 (18), 1668-1669 (1993).

Meltz, G., W. W. Morey y W. H. Glenn; Formation of Bragg gratings in optical fibers by a transverse holographic method, Opt. Lett. 14 (15), 823-825 (1989).

Mihailov, S. J., C. W. Smelser, D. Grobnic, R. B. Walker, P. Lu, H. Ding y J. Unruh; Bragg Gratings Written in All-SiO2 and Ge-Doped Core Fibers With 800-nm Femtosecond Radiation and a Phase Mask, IEEE J. Lightwave Technol. 22 (1), 94-100 (2004).

Nature Photonics 2 (3), 143-158 Technology Focus: Optical-fibre sensors (2008).

Othonos, A. y X. Lee; Novel and improved methods of writing Bragg gratings with phase masks, IEEE Photonics Tech. Lett. 7 (10), 1183-1185 (1995).

Othonos, A. y K. Kalli; Fiber Bragg Gratings: Fundamentals and Applications in Telecommunications and Sensing, Cap. 4, Artech House, New York, USA (1999).

Pérez-Millán, P., S. Torres-Peiró, J.L. Cruz y M.V. Andrés; Fabrication of chirped fiber Bragg gratings by simple combination of stretching movements, Opt. Fiber Technol. 14, 49-53 (2008).

Prakash, O., R. Mahakud, S. K. Dixit y U. Nundy; Effect of the spatial coherence of ultraviolet radiation $(255 \mathrm{~nm})$ on the fabrication efficiency of phase mask based fiber Bragg gratings, Opt. Comm. 263, 65-70 (2006).

Preciado, M. A., V. García-Muñoz y M. A. Muriel; Ultrafast all-optical Nth-order differentiator based on chirped fiber Bragg gratings , Opt. Express 15 (12), 7196-7201 (2007).

Russo, N. A. y R. Duchowicz; High Frequency Pulse Trains from a Self-Starting Additive Pulse Mode-Locked All-Fiber Laser, Opt. Comm. 283 (1), 113-117 (2010).

Russo, N. A., G. F. Arenas, S. B. Noriega y R. Duchowicz; Shrinkage measurement of BIS-GMA resins by embedded FBG. Trabajo presentado en VII Ibero-American Conference On Optics And X Latin-American Meeting On Optics, Laser And Applications (RIAO-OPTILAS 2010), Lima, Perú; 20 al 24 de Septiembre (2010).

Yeh, H. C., M. J. Shelton, Y. H. Tsang y T. A. King; Fabrication and characterization of fibre Bragg gratings for near $2 \mu$ m operation, Meas. Sci. Technol. 14 (10), 1747-1752 (2003).

Zagorul'ko, K. A., P.G. Kryukov, E.M. Dianov, A. Dragomir y D.N. Nikogosyan; Fibre-Bragg-grating writing in single-mode optical fibres by UV femtosecond pulses, IEEE J. Quantum Electron. 33 (8), 728-730 (2003). 